\title{
Gestión de calidad y su dimensión ético competitiva en la pequeña y mediana empresa metalmecánica de la región zuliana
}

\author{
Ferrer, Juliana * \\ Vázquez, José Luis ** \\ Clemenza, Caterina *** \\ Pérez, Rosario ****
}

\section{Resumen}

Este artículo tiene el propósito de estudiar las iniciativas que en materia de calidad y dimensión ético-competitiva, son asumidas por la pequeña y mediana empresa metalmecánica zuliana, y explorar además sus posibilidades de mejora y propuestas para estimular el crecimiento. Se aplicó una entrevista estructurada a 23 gerentes de empresas pequeñas y 13 de empresas medianas, ubicadas en el estado Zulia, utilizando muestreo estratificado con afijación proporcional. Los resultados de investigación muestran que las empresas no presentan una instalación formal del modelo de calidad de gestión, sólo elementos relacionados con la confiabilidad y apego a especificaciones, elementos del sistema que son generados por factores externos a la organización, representados ante todo por las exigencias formales asumidas por la actividad petrolera, su principal cliente. Además, en su mayoría no cuentan con herramientas estratégicas escritas que sirvan de guía para su desarrollo futuro, situación que aunada a la limitada internalización de valores éticos-competitivos representados por calidad, excelencia, confiabilidad, entre otros; generan escasas posibilidades para incorporarse de manera adecuada a una labor sinérgica, en función del crecimiento del sector. Se concluye que es necesario adelantar acciones de mejora dentro del sector estudiado, compatibles con el modo de vida de cada estrato en particular; lo que incluye entender sus formas de organización, desarrollar propuestas de cambio desde cada empresa, y en todas ellas, para que en conjunto puedan superar sus debilidades, y poder buscar formas estratégicas de convivencia que garanticen su perpetuidad dentro del sector.

Palabras clave: Gestión de calidad, mejora continua, cambio organizacional, dimensión éticocompetitiva, pequeña y mediana empresa metal-mecánica.

\section{Recibido: 01-11-25 . Aceptado: 02-03-04}

* Economista. Doctor en Ciencias Gerenciales. Maestría en Gerencia de Empresas. Investigadora del Instituto de Investigaciones de la Facultad de Ciencias Económicas y Sociales (FCES) de la Universidad del Zulia (LUZ). E-mail: jumferrer@cantv.net

** Economista. Maestría en Estadística e Investigación de Operaciones. Investigador del Centro de Estadística y de Operaciones de la FCES de LUZ.

*** Economista. Magíster en Gerencia de Empresas. Investigadora del Instituto de Investigaciones de la FCES de LUZ.

**** Candidato a Magíster en Gerencia de Empresas. FCES de LUZ. 


\section{Quality Management in Its Ethical-Competitive Dimension in the Small and Medium Sized Metalworking Industry in the Zulia Region}

\section{Abstract}

The purpose of this article is to study initiatives which, in the realm of the quality and ethical-competitive dimension, are undertaken by small and medium sized metalworking industries in Zulia State, and to explore the possibilities of improvement as well as for growth. A structured interview of 23 managers of small companies and 13 managers of medium sized companies located in Zulia state was undertaken utilizing a stratified sample with proportional representation. The results indicated that the companies studied do not have a formally installed management quality model, but only elements related to responsibility and compliance with specifications, elements of the system which are generated by external organizational factors, represented for the most part by formal specifications required by the oil industry, their principal client. Furthermore, the majority of the companies have no strategic tools with which to guide their future development, a situation which is compromised even more by their limited internalization of ethical-competitive values such as quality, excellence, and reliability, among others: and this generates little possibility for the adequate incorporation of labor synergy for the purpose of industry growth. In conclusion it is necessary to go ahead with actions which could improve this sector, and which would be compatible with the life styles of each stratum, and which would include understanding organizational forms and developing proposals for change in each company, so that together the sector can overcome its weaknesses and look for convenient strategies that would guarantee the continuity of the sector.

Key words: Quality management, continuous improvement, organizational change, the ethical-competitive dimension, small and medium-sized metal-working industry.

\section{Introducción}

Cuando se busca en la historia la palabra calidad es necesario remontarse a épocas verdaderamente antiguas. Sin embargo, es a comienzos del pasado siglo XX, a partir de los aportes de una gran cantidad de maestros de la administración, cuando se produce en el mundo el principio de una gran revolución en el área gerencial (Berry, 1996). Más tarde, durante la tercera década de este siglo, Shewhart (1939), sienta las bases de lo que más tarde se llamaría gestión de la calidad.

Las dos últimas décadas del siglo XX han sido testigos de cambios transcen- dentales en el entorno, que enfrentan las empresas, las cuales como producto de sus viejas formas de operar no funcionan en tiempos donde, la competitividad, los cambios en las relaciones laborales y la globalización, han exigido una nueva cultura a las organizaciones. Se requiere entonces, emprender cambios para adaptar sus estructuras y colocarlas en condiciones para operar en ese nuevo ambiente.

Como respuesta a tal necesidad, a partir de los años 80 el mundo occidental toma conciencia de la importancia de incorporar un nuevo concepto, gestión de la calidad y comienzan a adoptar una buena parte de las ideas y prácticas del 
control de calidad total, instituido por los japoneses al principio de la segunda mitad del siglo, con el propósito de producir los cambios que se requerían en esos países (Berry, 1996).

Hoy, son asumidos los conceptos de calidad, gestión de la calidad y calidad de servicios, como derivaciones de un sistema diferenciado por sus ámbitos, que abarca un conjunto de actividades y procesos adelantados por la empresa, asumiendo el énfasis por el cliente interno, externo y la mejora continua de los procesos (Clery, 2001).

En el caso particular de su aplicación al ámbito venezolano, esta nueva concepción no es más que el producto de esfuerzos aislados, que en términos de la filosofía de gestión de calidad, llevan adelante algunas empresas, con la finalidad de ofrecer mejores productos y servicios, pero sobre todo satisfacer las necesidades de confiabilidad asumidas por el cliente; elementos que no toman en cuenta la necesidad de internalizar esos rasgos, como filosofía ético competitiva.

Bajo tal realidad surge la inquietud por el desarrollo del presente artículo, el cual tiene por finalidad estudiar las iniciativas en torno a la gestión de la calidad y su dimensión ético-competitiva en la pequeña y mediana empresa metalmecánica zuliana. Para tal efecto y atendiendo a la clasificación de tamaño (OCEI, 1997), se asumió como universo objeto de estudio las 144 empresas que conforman el sector. En la recopilación de información se utilizó muestreo estratificado, con afijación proporcional, seleccionando 23 empresas pequeñas y 13 medianas.

Esta inquietud surge como un aporte a la necesidad perentoria de las empre- sas venezolanas de incorporarse a una mejora de sus procesos, en función de un crecimiento consolidado del sector industrial sujeto a estudio.

\section{Significado de calidad total}

Aproximarse a una definición precisa del término calidad es una tarea altamente complicada. Si se revisa la extensa bibliografía que al respecto existe se concluye que hay una gran variedad de significados para este término. Quizás la definición más usada, Adecuación al Uso (Juran, 1990), no aporta mayor claridad al respecto, ni establece bases para una aplicación práctica al concepto.

Deming (1986) define calidad como un sistema, libre de defectos, entendiendo defectos como todo aquel desperdicio que hace que el sistema, no funcione como debería ser y sea incapaz de satisfacer los requerimientos del cliente. Este autor añade al concepto de calidad un elemento que aporta grandes beneficios a la idea contemporánea: el proceso. Señala que para optimar los resultados, es necesario mejorar el proceso. Aunque la calidad es juzgada a partir de los resultados, la mejora de éstos y su adecuación a las necesidades actuales y potenciales del cliente, solo se logra concentrando la atención en los procesos.

En tanto, Cantun (1997), incorpora el concepto afirmando que no es más que una filosofía, una estrategia, un modelo de hacer negocios, siempre focalizado hacia el cliente. Este término no sólo se refiere al producto o servicio en sí, sino que es la mejoría permanente del aspecto organizacional-gerencial; tomando una organización como una máquina gi- 
gantesca, donde cada trabajador, gerente o funcionario del más bajo nivel jerárquico, están comprometidos con los objetivos empresariales.

Refiere Singh (1998), que el uso de la calidad total conlleva ventajas, pudiendo citar como importantes el hecho de ser potencialmente alcanzable si hay decisión del más alto nivel; igualmente, ella permite mejorar la relación del recurso humano con la dirección y sobre todo, reduce los costos aumentando la productividad, eficiencia y eficacia de la operación.

Para Cobra (2000), la definición del concepto de calidad, en pocas palabras, no es fácil. La empresa debe establecer requisitos y especificaciones que permitan fijar metas de calidad en los diversos niveles de la organización. La calidad es un concepto formulado a la luz de la percepción del consumidor y sólo puede definirse, con base en las especificaciones que satisfagan sus necesidades. En otros términos, el consumidor determina qué es la calidad.

A partir de la definición anterior una tendencia que pareciera contener elementos de considerable interés, es la que presenta a la calidad como el juicio que tienen los clientes o usuarios sobre un producto 0 servicio; es el punto en el que sienten que sobrepasa sus necesidades o expectativas (Gitlow, 1991). Esta definición presenta de manera apropiada componentes que permiten operacionalizar la definición de calidad, entre éstos, se pueden mencionar a los clientes con sus juicios, sus necesidades y sus expectativas; pero también hace énfasis en la necesidad de sobrepasar las expectativas del cliente, como una vía para mantener la competitividad.

Refiere Berry (1996), como una ventaja competitiva se logra cuando se analizan cuidadosa y constantemente las necesidades del cliente y cuando se organiza y trabaja para satisfacer tales necesidades la primera y todas las veces. Desde este punto de vista, es el cliente quien define la calidad del producto o servicio ofrecido.

Igualmente Clery (2001) afirma, como la calidad total es un sistema de gestión que abarca a todas las actividades y a todas las realizaciones de la empresa, poniendo especial énfasis en el cliente interno y en la mejora continua.

En tanto Cobra (2000), establece que la calidad es un elemento importante para definir un servicio ofrecido, ya que con base en este criterio, el consumidor establece la diferencia entre el servicio de una empresa y el de sus competidores.

Por tanto, hoy día calidad significa algo más que confiabilidad del producto; hoy día significa un esfuerzo para lograr la compatibilidad en términos de Calidad Total, es decir, un esfuerzo conjunto, en el cual participan todos los miembros y todas las funciones en una organización (Singh, 1998).

La compatibilidad en términos de la calidad total se enfocará en el mejoramiento de productos, servicios y procesos; cuando mejoren, causarán un impacto en la productividad, la satisfacción del cliente y las utilidades. Ese impacto se verá tanto interna como externamente (Ver Diagrama 1). Un aumento de la calidad mejora la satisfacción del cliente, puesto que le otorga al producto o servicio un valor agregado además, ayuda a cubrir y superar expectativas enunciadas o implícitas en los requerimientos de éste. A su vez, un aumento de esa calidad mejora la productividad por la vía de la 


\section{Diagrama 1 Compatibilidad en términos de la calidad total}

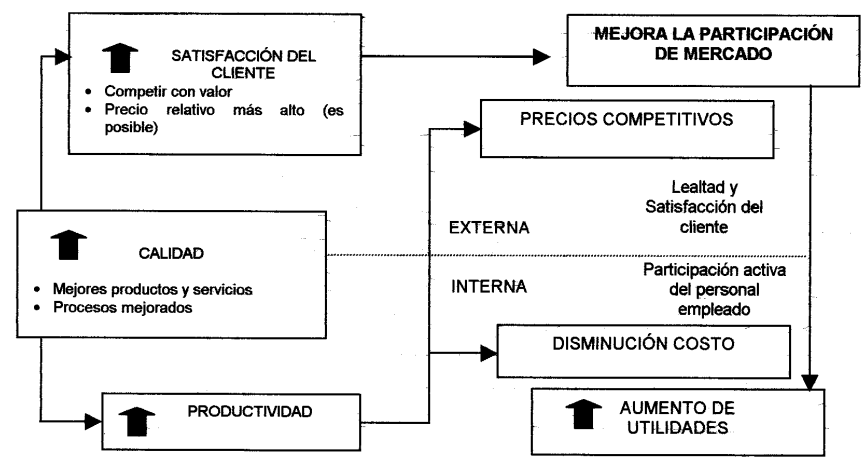

Fuente: Adaptación de Singh, 1998.

disminución de los errores, en los productos y servicios y la disminución de los reprocesos; situación que se refleja en precios competitivos, sobre la base de menores costos de producción o prestación del servicio. Una mayor satisfacción del cliente y unos precios competitivos originan una mejor participación del mercado, lo cual obviamente genera un aumento en la utilidad de la empresa.

Singh (1998), estudia el ambiente interno y externo de la organización y refiere cómo tal compatibilidad se logra internamente mejorando la calidad y productividad, lo que permitirá bajar precios, incrementar la participación de mercado y obtener mayores utilidades; bajo esta forma alternativa, el costo más bajo proporciona un incremento directo en las utilidades. En tanto, el impacto externo, se logra a través de un nivel más elevado de calidad que permita aumentar la satisfacción del cliente, incrementar su lealtad y lograr el posicionamiento.
De allí la importancia del significado de la calidad total visto como una dimensión de interés para la empresa, al representar una ventaja competitiva en el mercado de productos y servicios, explicada por el conjunto de actividades que de manera cuidadosa y constante atiende a las necesidades de un cliente externo, otorgando además un énfasis particular al cliente interno y a la mejora continua.

\section{Gestión de calidad}

La mayoría de los gerentes no dudan en aceptar la necesidad de un cambio en un mundo de aceleradas transformaciones, e incluso parecieran comprender instintivamente los principios que fundamentan la nueva visión de la calidad. Es cuestión de sentido común que el cliente determine la calidad; por lo que es necesario investigar sus necesidades, luego interpretar la información recogida y ayudar a los miembros de la organización a realizar un trabajo, de manera de 
lograr un producto o servicio que satisfaga las necesidades y expectativas detectadas. Nada más lejos de la realidad, que el asumir lo que el cliente necesita. En este sentido viejas reglas están enraizadas en las estructuras de las organizaciones, por lo que el cambio no es tan fácil como parece; se explica entonces, la gran cantidad de fracasos ocurridos al iniciarse el proceso de cambio (Hammer y Champy, 1997).

Sin embargo, hoy existen empresas exitosas en la instrumentación del cambio, generándose como consecuencia, numerosos estudios en cuanto a los elementos que han caracterizado el éxito. Atendiendo a esta inquietud Serieyx (1991) en función de estas experiencias propone una metamorfosis por la que debe pasar una empresa para este cambio radical, representadas por: Un interés mayor de la empresa por su mercado, que por sí misma; una relación con el cliente oportuna, una alianza directa con el proveedor, entre otras; esto con la finalidad de orientar el pensamiento hacia el proceso; abandonar el modelo de producción-inspección y tomar el de producir bien, desde la primera vez. Por ello se habla de sustituir el modelo mecanicista por un modelo, donde los componentes asumen misiones y trabajan en equipo; armonizando relaciones con todos los elementos del sistema, incluyendo a los proveedores; y por tanto prefiriendo la prevención por encima del control; abandonando así el despilfarro proveniente de la duplicación y del trabajo innecesario.

En ese mismo sentido Harrington (1988) establece un conjunto de directrices básicas, entre las que se pueden mencionar, el compromiso y la participación de la alta dirección, puesto que sólo así la instalación del modelo de calidad sería exitoso. Afirma que la participación total de la administración es vital dentro de esta iniciativa ya que la calidad es un problema de todo el sistema; es decir, el entrenamiento e inducción en la estructura jerárquica con el fin de incorporar los beneficios del modelaje; la participación de los empleados en la toma de decisiones con el fin de lograr un clima organizacional que estimule la creatividad de todos los participantes en la organización; la participación de los proveedores, ya que sólo con unas exitosas relaciones con el proveedor se pueden obtenerse ventajas competitivas; y sobre todo un sistema de reconocimiento que estimule y refuerce el mejoramiento al desempeño.

En tanto Berry (1996), habla de una serie de obstáculos que pudieran eventualmente perjudicar el proceso de cambio. Alguno de ellos son, el enfoque de corto plazo; la falta de comunicación en la organización; relacionar aumento de calidad con reducción de personal; delegar la responsabilidad sobre la calidad; buscar la mejora sólo en las personas y no en el sistema; restar importancia a la capacitación formal o informal; atribuir al departamento de calidad toda la responsabilidad de la calidad y sobre todo, construir una estructura de calidad aparte de la propia estructura organizacional.

Igualmente Scholtes (1993) indica una serie de principios claves para la instalación de la nueva filosofía, destacando como vitales, los clientes y sus necesidades como la razón de ser de la organización; la calidad de los productos y servicios como resultante de sistemas, procesos y métodos de calidad, por lo que los 
gerentes deben comprender a éstos en cuanto a capacidad y variación; toda la organización debe enfocarse en la calidad; los logros en calidad de la organización deben basarse en la capacidad de manejar los métodos de mejoramiento; debe privilegiarse el abandono de la complejidad y debe acogerse la simplicidad; $y$ finalmente, debe instalarse una nueva forma de liderazgo.

Como puede observarse, son muchos los elementos clave visualizados para el proceso de cambio. Sin embargo, existen algunos que son comunes a todos los planteamientos. Entre ellos vale la pena mencionar, el conocimiento de las necesidades de los clientes, la comprensión de la variación por parte de quienes gobiernan el sistema, el pensamiento orientado hacia los procesos y el manejo de la complejidad. Aunque están altamente interrelacionados, debe hacerse una reflexión sobre ellos a fin de conocer como funcionan y como deben enfrentarse para encaminar la organización hacia el cambio necesario.

\section{Calidad de servicios. Una dimensión vital de la gestión de la calidad}

La década de los ochenta estuvo marcada por el creciente descontento de los clientes respecto a la calidad, tanto de productos como de servicios. Muchos de los problemas con los productos ofrecidos estaban centrados en el mal servicio en el punto de compra, incluyendo las dificultades para resolver los problemas de fábrica, para la obtención de reembolsos y para lograr que se atendiera el servicio postventa. Así, especialmente las indus- trias de servicio eran criticadas tanto por las llamadas fallas humanas de los empleados, como por los errores en los aspectos técnicos y de mercadeo, en la entrega del servicio (Lovelock, 1997).

Refiere Cobra (2000) como la calidad de un servicio tiene dos dimensiones: Una instrumental que describe los aspectos físicos del servicio, y otra relacionada con las características funcionales, que describe los aspectos intangibles o psicológicos del servicio. En consecuencia, un servicio se identifica por los aspectos "técnicos" y "funcionales", dos componentes fundamentales de la calidad. Por ello, dado que un servicio se consume en el mismo momento en que se produce, los aspectos técnicos y funcionales deben estar bien articulados para que proporcionen al consumidor una percepción objetiva de la calidad.

Según Berry (1996), calidad en tal sentido, abarca el grado de conformidad con los requerimientos y adecuación al uso; es decir, la satisfacción con las características que conforman productos y servicios en relación con las expectativas de los clientes.

Desde esta perspectiva, se considera que se logra la calidad en el servicio cuando existe compatibilidad entre las expectativas del segmento de clientela y el servicio prestado por la organización. Por lo tanto, la calidad del servicio se traduce en lo que desea el cliente; es decir, su percepción acerca del mismo, lo cual es pertinente para la empresa.

Basado en la satisfacción del cliente Horovitz (1991) agrega, que la calidad de servicio es el nivel de excelencia que la empresa ha escogido alcanzar, para satisfacer a su mercado meta. Refiere 
como la calidad del servicio representa al mismo tiempo, la medida en que se logra dicha calidad; es decir, satisfacer las especificaciones de acuerdo a los requerimientos del mercado objetivo, respondiendo así a necesidades que tiene el consumidor, a través de todo el proceso de compra, entendiéndose por éste, desde la decisión de compra hasta las sensaciones posteriores al uso del servicio.

Entonces, la calidad del servicio corresponde al grado de satisfacción que experimenta el cliente o consumidor final por la forma en que percibe fue atendido por la organización, la efectividad del servicio que recibió, desde que hizo el primer contacto, hasta el tratamiento post-venta y por la forma en que recibió dicho servicio (Lovelock, 1997).

Horovitz (1991) agrega, que las grandes organizaciones de servicios del mundo han concentrado sus esfuerzos en el desarrollo del factor humano, buscando establecer la excelencia en el servicio que prestan, como el elemento clave y diferenciador en cada uno de sus mercados.

Por su parte Bradford y Albrecht (1992) define la calidad del servicio como las características, actos e información que aumentan la capacidad del cliente para materializar el potencial de un servicio esencial enfocándose en la interacción verbal y no verbal de los clientes con la empresa.

Asimismo, Ciampa (1992), coincide con lo planteado, al referir que la calidad del servicio se define, como las acciones que se realizan en la búsqueda de la satisfacción de las expectativas y necesidades del cliente. Esta definición se enfoca sobre los objetivos cuantitativos que se establecen y de acuerdo a los cuales, se hacen las comparaciones para observar el avance de los objetivos organizacionales; lo que supone un análisis de las condiciones que rodean el servicio y la percepción que tiene el cliente sobre el mismo.

Desde la perspectiva de Shaw (1997) el cliente desea calidad de productos y servicios, que no es más que ver satisfechos sus requerimientos y necesidades a través de un proceso lógico que alcanza resultados específicos. La calidad implica eliminar fallas cualitativas y cuantitativas; agrega que las empresas las permiten porque no están conscientes de los costos, ni de los niveles "aceptables" de las mismas, para las organizaciones.

Refieren algunos autores (Ciampa, 1992; Shaw, 1997) como las fallas no satisfacen las necesidades de los clientes y crean una imagen de baja calidad en la mente del mercado, por lo cual es esencial que las organizaciones no sólo se enfoquen a monitorear el mercado, sino a autodiagnosticarse para determinar cómo están internamente ejecutando sus acciones. De allí que es esencial conocer la opinión tanto de los clientes internos, como la percepción de los clientes externos.

Lovelock (1997), argumenta que debido a la naturaleza intangible y de múltiples facetas de muchos servicios, puede resultar más difícil evaluar la calidad de un servicio que la calidad de un bien; tal argumento se evidencia en que los clientes a menudo se involucran en la producción del servicio, por lo que es necesario trazar una distinción entre el proceso del servicio y el resultado real del servicio. Es decir, la calidad percibida de un servicio será el resultado de un proceso de evaluación, en el cual los clientes 
comparan sus percepciones de la prestación del servicio y su resultado con lo que ellos esperaban.

Así, la calidad de servicio se consolida en cinco vastas dimensiones (Lovelock, 1997): Tangibles (apariencia de los elementos físicos); Confiabilidad (un desempeño confiable y preciso); Actitud responsiva (prontitud y espíritu servicial); Garantía (competencia, cortesía, credibilidad y seguridad) y Empatía (acceso fácil, buenas comunicaciones y comprensión del cliente).

Al analizar las dimensiones del servicio, Cobra y Zwarg (1991) refieren como en el proceso de servicio existen elementos que son tangibles e intangibles. Además entre las características de los servicios destacan la intangibilidad, pues los mismos son el resultado de un esfuerzo o acción donde son poseídos pero no consumidos. Sin embargo, a pesar de no poder ser palpados, si es posible observarlos a través de la infraestructura material que rodea al servicio.

Por otra parte Bradford y Albrecht (1992) señalan, que existen diversas áreas involucradas en el proceso de prestación de un servicio de excelencia. Aseveran que para prestar un servicio a los clientes, se debe tomar en cuenta a quién se quiere satisfacer, teniendo en cuenta las expectativas del cliente, por lo que hay que tratar de conocerle mejor e indagar sobre el mismo; por tanto se recomienda ofrecerle un servicio personalizado para mejorar su calidad de vida, involucrando aspectos técnicos que apoyen el cumplimiento de los ofrecimientos y compromisos.
Desde la perspectiva de Kotler y Armstrong (1996), el propósito de toda empresa de servicio es descubrir, cuáles son los factores críticos y determinantes, que utilizan los clientes para definir la calidad; es decir, indagar lo que es realmente importante para ellos.

Destacan cinco dimensiones principales a través de las cuales los clientes evalúan al servicio:

- La Confiabilidad o, habilidad que posee la organización para suministrar el servicio prometido de forma confiable, seguro y cuidadoso;

- La Variabilidad del servicio o, capacidad de la empresa para ofrecer a sus clientes diversidad o modalidades del servicio para dar cobertura a sus requerimientos y necesidades;

- La Tangibilidad o, aquellos productos físicos que intervienen en la prestación del servicio;

- La Capacidad de respuesta o, actitud que la organización ofrece a los clientes de modo que se preste un servicio oportuno;

- La Empatía 0, accesibilidad, comunicación y entendimiento para con el cliente.

Por su parte, Eiglier y Langeard (1989), consideran que la calidad de servicio debe evaluarse en tres dimensiones diferentes: el output, los elementos de la prestación del servicio y el proceso en sí mismo; en efecto, los tres son percibidos y demandados por el cliente.

Estas tres dimensiones de calidad global del servicio forman el marco estructural en el que pueden inscribirse las acciones de la empresa de servicio a favor de la calidad esperada. 


\section{Calidad como elemento ético-competitivo de la empresa}

Existen particulares características que frenan el desarrollo en el mundo competitivo y que son dignas de mencionar: Las fuentes convencionales de la ventaja competitiva, la administración tradicional, la rezagada actitud mental que no valora la flexibilidad y propensión al cambio y sobre todo, la poca capacidad de interrelación congruente entre organizaciones; son hechos que han generado a menudo condiciones de inercia y paralización frente a las oportunidades y desafíos, evidenciados en el ambiente del mundo global (Hit et al, 1999). Tal necesidad ha motivado que muchas empresas comiencen a pensar que sus posibilidades de éxito futuro dependen de una identidad, dispuesta a aceptar claras responsabilidades sociales y tomar decisiones congruentes dentro de un contexto donde las consecuencias aceptadas determinan un hecho moral, a partir de las demandas del entorno sociocultural. Bajo este contexto una ética de la empresa deberá contar con un sistema de valores que aflore de la cultura corporativa, los cuales deben estar alineados a través o a lo largo de la organización.

Cortina, et al (1997) justifican la necesidad de asumir por parte de la empresa el elemento ético correlacionado con el elemento competitivo a través de valores organizacionales como la calidad e innovación que unidos con la credibilidad generan la seguridad y confianza necesaria para los espacios sin fronteras donde transitan las empresas de hoy.
A innumerables organizaciones en el mundo les urge incrementar su competitividad, por lo que buscan estándares óptimos y vinculación en los procesos inmersos en la organización, que les permita interactuar con clientes internos y externos, gestionando así la mejora de los mismos, tal proceso requiere como elemento vital la acción diaria pero congruente de la empresa, a partir de una relación transparente con dichos clientes, potenciadas por dimensiones que garanticen aspectos tangibles e intangibles o psicológicos del servicio prestado.

La gestión de la calidad proporciona principios que permiten lograr una continua revisión de los estándares alcanzados con el fin de garantizar esa competitividad, destacando, como formas dinámicas: la calidad del producto y servicio como función directa del valor; la participación activa de clientes, proveedores y competidores como formas de generar mercados congruentes, pero sobre todo la aptitud y talento para competir dentro de la particular interconexión que exigen los mercados globales (Etkin, 1996; Fea, 1993; Cantun, 1997); fuente de cohesión, competitividad y calidad total.

Desde esta perspectiva el concepto de competitividad ha adquirido de alguna manera muchas y diversas interpretaciones. Así Romero, et al (1997) la refiere como la capacidad que tiene la empresa para obtener una posición ventajosa en el mercado interno y ofrecer de alguna manera capacidad para brindar calidad y productividad para enfrentarse a los mercados globalizados.

Para Etkin (1996), la competitividad implica una actitud particular, estado o atributo de la empresa o modo de 
funcionamiento congruente con la realidad de mercados abiertos, donde hay que enfrentar amenazas y desafíos cotidianos.

Ferrer (2001) interpreta la competitividad, como un atributo de la empresa donde participan clientes internos y externos, en la búsqueda de las congruencias de los mercados donde transita. Tal congruencia se aborda a través de su actitud para manejar los factores involucrados con las variables controlables por la empresa, como el producto y el servicio de postventa; pero también por la posibilidad de mitigar variables no controlables, representadas entre otras por, el desarrollo de tecnologías, regulaciones internas o cualquier movimiento de los competidores que no atienda a una interconexión propicia para transitar en espacios marcados por la incertidumbre de hoy.

En este mismo sentido Cortina (1998) incorpora el concepto de competitividad a través de la categoría ética empresarial, destacando la idea de que las empresas que se conducen éticamente terminan siendo competitivas, poniendo énfasis en variables como calidad e innovación, pero también credibilidad al interactuar en mercados globales.

Cortina, et al (1997; 1998), afirma además, que por lo general se tiene la sensación de que existe una incompatibilidad entre ética y competitividad; dado que se asume está última, como sinónimo de desalojar al adversario y, por tanto, no parece que pueda compaginarse con una conducta ética. Sin embargo, estudios realizados desde finales de los ochenta (Cortina 1985; 1990; 1992; 1993; 1996; Cortina, et al, 1997) han demostrado que existe una correlación positiva en- tre ambas dimensiones, aunque una empresa ética, siempre es más competitiva; entendiendo por competitividad el deseo de permanecer en el mercado a largo plazo con un beneficio suficiente que le permita perdurar.

Además, refiere que las empresas son competitivas por dos razones: merecen credibilidad y generan confianza. La credibilidad es un valor que lleva a la empresa a generar seguridad y confianza, siempre que no se asuma relatividad en el resto de los valores organizacionales asumidos (Ferrer, 2001); el segundo gran valor es la innovación, elemento de la creatividad, implícito en la calidad y gestión de calidad.

Por su parte la gestión de la calidad conlleva a generar ventajas competitivas, y además, a fomentar con un poco de imaginación y racionalidad la apertura de mercados. Estos últimos elementos unidos a la credibilidad y la innovación, favorecen no sólo la permanencia en el tiempo, sino la garantía de rentabilidad en los mercados, sin tener que obviar la lógica empresarial (Cortina, 1998; Ferrer, 2001).

\section{La Pequeña y Mediana Empresa y sus iniciativas frente a la de gestión de calidad}

En la última década, ha habido un creciente interés en la pequeña y mediana empresa como un vehículo idóneo para el crecimiento económico de los países subdesarrollados y particularmente, los latinoamericanos. Igual acontecer se observa en los países industrializados, donde la participación de las pequeñas empresas en la creación de empleo y de 
valor añadido ha sido creciente, incorporándose efectivamente en la producción de bienes y servicios, aprovechando cambios en la organización productiva, modificaciones de la demanda y las facilidades que brinda la tecnología informática (Lanusse, 2001).

El colapso de las estructuras tradicionales de organización, puesto de manifiesto en las reducciones de tamaño, la subcontratación, las alianzas estratégicas, la reingeniería de procesos, la gestión de la calidad y los equipos virtuales o dispersos geográficamente, evidencian fuerzas tanto en el sector público como en el privado que tienden a aplanar y comprimir organizaciones para que cuesten menos y funcionen mejor (Lanusse, 2001; Rosales, 1996).

Si a lo anterior se agrega el concepto de competitividad y se entiende el mismo como el resultado del aporte de las empresas individuales, dentro de redes de procesos de producción, en las que se evidencia una significativa participación que en ellas tienen la pequeña y mediana empresa, para las diferentes etapas de procesos relacionados con el suministro, producción, mercadeo y servicio de postventa, se evidencia entonces su relevancia al momento de evaluarlas dentro del contexto de cualquier economía globalizada (Rosales, 1996; Ferrer, 2001).

Hoy la naturaleza de las relaciones y no el tamaño es el factor competitivo clave. La interacción entre cliente internos y externos para lo cual la gestión de la calidad se convierte en una herramienta idónea, representa un elemento crucial en la interacción entre las empresas bajo el ámbito de los mercados nacionales e internacionales (Lanusse, 2001).
En el contexto venezolano, la pequeña y mediana empresa, ha tenido un papel importante en el desarrollo económico del sector industrial, al producir ciertos bienes que las grandes empresas no están dispuestas a producir por la baja demanda en el mercado. Además, la producción en la pequeña y mediana empresa requiere el uso intensivo de mano de obra, razón por la cual el desarrollo de este segmento determina en buena medida los niveles de empleo y bienestar en el país, no siendo este aspecto el único en el cual se manifiesta la contribución de dicho sector (Clemenza, et al, 1997).

Sin embargo, dentro de este contexto organizativo de la pequeña y mediana empresa en general, existen insuficiencias gerenciales, organizativas y técnicas, que impiden hacer uso óptimo de las capacidades humanas y de equipamiento disponibles, para mejorar la productividad e incentivar el desarrollo de cambios tecnológicos y organizativos. El sector de la pequeña y mediana empresa metalmecánica no ha escapado a esta problemática, que afecta a todos los estratos y sectores de actividad del país. En lo relativo al mercado, la constante escasez de demanda frente a las potencialidades de la oferta, ha generado excedentes de capacidad instalada y una progresiva contracción en el nivel de empleo. En cuanto a la oferta, los problemas típicos son los crecientes costos energéticos y laborales, el valor de la materia prima y los gastos financieros (Romero, et al, 1997; Clemenza, et al, 1997).

Por ello, la tendencia a establecer alianzas estratégicas para la innovación, implica una excelente oportunidad para las pequeñas y medianas empresas, las cua- 
les pueden insertarse en las estrategias de innovación global, siempre y cuando sean capaces de desarrollar la habilidad de innovar y una destreza que le permita negociar bajo el contexto de la cooperación. (Rosales, 1996; Ferrer, et al, 1999).

Puede afirmarse que hoy, existe a nivel mundial una tendencia orientadora hacia el rescate de las pequeñas y medianas empresas, con el propósito central de disminuir las desventajas de carácter competitivo, respecto a la gran empresa (Romero, et al, 1997). Las medidas de estímulo incluyen asistencia técnica, financiera, fiscal, así como el subsidio para el desarrollo e investigación en nuevas tecnologías y para el entrenamiento gerencial, como forma de garantizar un espacio para estructuras, que representan hoy, la fuente de empleo de un porcentaje significativo de la población mundial, además de ser responsables de la producción de insumos, partes y piezas de la gran empresa, en el contexto de los mercados globalizados (Hernández, 1998; Romero, et al, 1998).

Así, dichas empresas han adquirido gran importancia, tanto en el entorno nacional como el internacional, para el desarrollo económico y social de los países. La estrategia de crecimiento basada en el liderazgo del Estado y los grandes proyectos industriales han demostrado no ser tan efectiva para conducir los países al desarrollo; por eso es que ahora se está dirigiendo la atención hacia las posibilidades que muestran las pequeñas y medianas empresas como motor de un nuevo desarrollo más equitativo (Lanusse, 2001). La relevancia adquirida por la pequeña y mediana industria en los últimos años, subyace también en la redi- mensión de las grandes empresas, producto del acelerado cambio tecnológico, en pequeñas unidades estratégicas de negocios, las cuales al especializarse pueden reunir las condiciones para alcanzar mayor eficiencia competitiva y flexibilidad (FEDEINDUSTRIA, 1996).

Las pequeñas y medianas empresas, son un factor clave para las políticas y estrategias de desarrollo nacional, y contribuyen a escala mundial con más de $60 \%$ del empleo y más del $40 \%$ del valor agregado, pues favorecen la absorción de mano de obra no calificada, son fuente de empleo, contribuyen a la descentralización y potencian la exportación de productos con mayor valor agregado, entre otros aspectos (FEDEINDUSTRIA, 1996).

Pilleti (1999), refiere como en los últimos años ha ocurrido un fenómeno que le ha dado fuerza e importancia a la pequeña y mediana empresa, relacionado con los cierres de producción en ciertos rubros que ha hecho la gran industria para poder sobrevivir, en términos de su tamaño, tipos de productos, áreas, personal y la contratación externa de las labores que están íntimamente relacionadas con la actividad que adelanta. De esta forma han surgido pequeñas y medianas industrias dispuestas a satisfacer necesidades en diferentes campos las cuales han representado una respuesta oportuna para productos y servicios de bajo interés para empresas de gran envergadura.

Aunado al fenómeno de la globalización, el mundo se encuentra frente a una nueva gran oportunidad para la pequeña y mediana empresa. Los cambios que se están suscitando las enfrentan a un proceso de segmentación de los mercados, en contraposición de los merca- 
dos masivos que habían prevalecido tradicionalmente (Pizani, 1994).

\section{Algunas características de la Pequeña y Mediana Empresa Metalmecánica Zuliana}

La pequeña y mediana empresa metalmecánica zuliana representa un soporte para todas las grandes empresas existentes tanto en la región como en el país, especialmente como suplidora de partes, piezas y servicios para las empresas petroleras.

La industria metalmecánica abarca todos aquellos sectores productivos que se dedican a la transformación de metales. Incluye tanto a las plantas de fundición y forja como los talleres de estampado, corte y soldadura, tratamiento térmico, de metales diversos; agrupa los establecimientos de armado y ensamblaje final de maquinaria eléctrica y no eléctrica, de vehículos y materiales de transporte y de equipos varios de índole científica y adicionalmente, todos los servicios relativos a mantenimiento y reparación de estructuras y equipos mecánicos (Katz, 1983; Pilleti, 1999).

Dos hechos han producido el desarrollo de éste sector económico en la región: La influencia de la actividad petrolera y la política de sustitución de importaciones; en la actualidad puede afirmarse que el Zulia cuenta con una industria perfeccionada, con unos estándares de calidad moderados, por cuanto la misma nació y se hizo fuerte a través del suministro de bienes, equipos, partes y piezas destinadas al sector petrolero (Ferrer, et al, 1999).
Para efectos de analizar la pequeña y mediana empresa metalmecánica se asumió una composición de la muestra, representada en un $63,88 \%$ de empresas pequeñas y $36,12 \%$ de empresas medianas; de ellas el $78,60 \%$ se clasifican como pertenecientes al grupo de fabricantes de productos metálicos y el $21,40 \%$ como proveedores de servicios, considerando la Clasificación por Actividad Económica de la OCEI (1997). Del total de empresas recogidas en la muestra, el 92,90\% utilizan capital de origen nacional; vale la pena destacar que en este grupo se encuentran todas las empresas pequeñas y el $66,70 \%$ de las medianas, el resto, $7,10 \%$ del total y $33,30 \%$ de la empresas medianas utilizan capital mixto.

Los resultados arrojados por la encuesta muestran que las empresas trabajan con una alta capacidad ociosa. Se puede observar que el $76,90 \%$ de las empresas trabajan por debajo del $50,00 \%$ de su capacidad, notándose que la empresas pequeñas $(81,90 \%)$ tienen una situación más crítica que la empresa mediana $(50,00 \%)$.

Por otro lado, el $85,70 \%$ de las empresas trabajan un solo turno, destacándose que casi la totalidad de las empresas pequeñas $(90,90 \%)$ presentan esa característica, mientras que el $66,70 \%$ de las medianas se encuentran en situación similar. Es relevante afirmar que esta alta propensión a trabajar un solo turno por parte de las empresas del sector está asociado con el alto índice de capacidad ociosa que ellas presentan, que pudiera explicarse por la fuerte contracción que ha sufrido la demanda de los productos del sector metalmecánico, debido a la reducción de la actividad petrolera que se ha manifestado en los últimos años. 
El 7,10\% de las empresas refirió no tener competidores, todas ellas empresas medianas, en tanto el $66,70 \%$ de las empresas medianas refirió tener competidores directos. En el caso de las pequeñas el $100 \%$ considera que trabaja en un mercado competido. Esto revela una fortaleza relativa, en las empresas medianas, ya que un número significativo de ellas no tienen competidores directos.

Por otro lado, la gran mayoría de las empresas pequeñas y medianas del sector producen con base en pedidos, característica que está muy asociada al desenvolvimiento del mercado y la tecnología del sector. Sólo algunos casos producen en grandes lotes y en pequeños lotes, $7,10 \%$ y $14,30 \%$, respectivamente, todos ellos perteneciente al grupo de la pequeña empresa.

La mayor parte de las empresas del sector son especializadas $(71,40 \%)$, correspondientes a un $63,60 \%$ de las pequeñas y la totalidad de las medianas. Esto revela una gran fortaleza de estas empresas debido al dominio tecnológico que esta alta especialización provoca, pero a su vez, en momentos de contracción de la demanda, esta característica puede convertirse en una gran debilidad, al poner a depender a la empresa de pocos compradores, con lo que su poder de negociación se reduce en gran medida (Ferrer, et al, 1999) .

Sólo el $28,60 \%$ realiza actividades de desarrollo tecnológico, existiendo una gran diferencia entre las pequeñas y las medianas, con un $18,20 \%$ y un $66,70 \%$, respectivamente. De allí se desprende una gran debilidad de las empresas pequeñas que les impide enfrentar con éxito el mundo competitivo que tiene ante si, por lo que, de no cambiar esta situación, deberá seguir con una estrategia de supervivencia, que a la larga no garantiza, su perdurabilidad (Ferrer, 2001). En el caso de la mediana se nota una mayor preparación para encarar las condiciones difíciles de un mundo globalizado. Esta situación se acentúa aún más cuando se observa que ninguna empresa pequeña tiene o está tramitando el certificado de ISO 9000 o ISO 14000, mientras que el $66,70 \%$ de las medianas están en vía de obtenerlo o ya lo tienen.

Otro factor que coloca en situación desventajosa a las empresas pequeñas lo constituyen el hecho que usualmente el propietario, que por lo general no tiene otra capacitación que la experiencia que haya podido acumular, es quien realiza el rol de gerente. En el caso de la mayoría de las medianas, este papel lo desempeña una persona contratada para tal fin $(66,70 \%$ del total de empresas medianas). Es indudable que esta persona cuenta, en la mayoría de los casos, con un mejor perfil que le permite enfrentar el entorno cambiante, altamente competitivo y globalizado que rodea a las empresas de este sector (Ferrer, et al, 1999).

\section{Correlación no paramétrica para los elementos éticos competitivos en la Pequeña y Mediana Empresa Metalmecánica}

Para los efectos de determinar la correlación entre los elementos competitivos y éticos se pudo constatar que existe una alta relación entre la competitividad y algunos elementos éticos, en especial el rasgo calidad, si sobre todo se considera 
que aquellos elementos de la competitividad ligados a este rasgo están íntimamente asociados a la situación competitiva de las empresas medianas y grandes. Para Cortina (1998), las empresas que se conducen éticamente terminan siendo competitivas. Refiere como en una primera aproximación a las variables se tiene la sensación de que existe incompatibilidad entre ambas y por tanto no pueden interactuar. Tal idea, surge de la apreciación de competitividad como sinónimo de desalojar al adversario; sin embargo, ambas dimensiones se correlacionan desde la perspectiva de entender a la competitividad como el deseo de permanecer en el mercado a largo plazo con un beneficio suficiente que le permita ser durable.

En este sentido, una empresa competitiva es aquella que tiene una actitud particular para manejarse en el mercado de manera congruente, mereciendo credibilidad y generando confianza, dos valores que unidos a la innovación y la creatividad implícita en la calidad coadyuvan a generar ventajas competitivas y traspasar fronteras de racionalidad en mercados inciertos. Señala Cortina (1998) que la credibilidad e innovación favorecen la supervivencia de la empresa y su prosperidad, sin tener que salir de la lógica empresarial del mercado.

Así al aplicar el coeficiente de correlación Rho de Spearman para variables no paramétricas, puede observarse que aquellas empresas que manifiestan valores como eficiencia, innovación, calidad, excelencia y éxito, han logrado a lo largo del tiempo, condiciones ligadas al potencial competitivo como el desarrollo de programas de productividad, ligadas a las actividades de desarrollo tecnológico (Rho $=0,849)$ y, conectados con progra- mas de calidad como el ISO 9000 , ISO $14000($ Rho $=0,645)$.

Así mismo es interesante destacar que un $76,90 \%$ de las empresas medianas, poseen misión, visión y código de ética escritos; además están conscientes de la necesidad de internalizar valores a compartir con clientes internos y externos (Rp $=0,645$ ) situación indispensable para el desarrollo de cualquier estrategia, en torno a conducir los destinos de la empresa.

Sin embargo, cuando desagregamos entre la pequeña y mediana empresa, puede visualizarse que la pequeña no posee condiciones para enfrentar situaciones de futuro, dado que sus habilidades competitivas son bajas; visualizado en el hecho de que sólo un $13 \%$ poseen misión y visión, no se tienen internalizado los valores que reconoce el gerente y éste, no posee herramientas estratégicas, ni operativas, que les permitan salir de las condiciones de subsistencia que la caracterizan.

En la Tabla № 1 se observan las condiciones calculadas utilizando la Rho de Spearman entre variables relacionadas con la competitividad y las relacionadas con el componente ético, asumidas a partir del rasgo calidad.

De su análisis se desprende lo siguiente:

- Variables tales como nivel de especialización y actividades de desarrollo tecnológico, no mostraron una asociación significativa respecto a los rasgos vinculados al componente ético. De tales resultados podría inferirse, una falta de coherencia entre los rasgos expresados como importantes en la empresa para fines estratégicos y la actividad que en realidad desarrollan y la forma como la ejecutan. 
Tabla 1

Pequeña y Mediana Empresa Metalmecánica.

Correlaciones no paramétricas entre los rasgos de competitividad y los rasgos vinculados al componente ético

\begin{tabular}{|c|c|c|c|c|c|c|c|}
\hline $\begin{array}{r}\text { Rasgos vinculados al } \\
\text { componente ético }\end{array}$ & $\begin{array}{l}\text { Misión } \\
\text { Escrita }\end{array}$ & $\begin{array}{l}\text { Visión } \\
\text { Escrita }\end{array}$ & $\begin{array}{c}\text { Reconocimiento } \\
\text { de Clientes } \\
\text { Internos y Externos }\end{array}$ & $\begin{array}{l}\text { Código } \\
\text { de Ética } \\
\text { Escrito }\end{array}$ & $\begin{array}{c}\text { Calidad y } \\
\text { Excelencia }\end{array}$ & Innovación & Confiabilidad \\
\hline Competitividad & \multicolumn{7}{|c|}{ Rho de Spearman } \\
\hline $\begin{array}{c}\text { Tecnología para el principal } \\
\text { producto o servicio }\end{array}$ & -0.328 & -0.328 & -0.325 & $0.519^{*}$ & 0.212 & 0.192 & 0.490 \\
\hline Nivel de Especialización & 0.400 & 0.400 & 0.396 & -0.278 & -0.418 & 0.192 & 0.490 \\
\hline $\begin{array}{c}\text { Actividades de Desarrollo } \\
\text { Tecnológico }\end{array}$ & 0.300 & 0.300 & 0.346 & -0.325 & -0.209 & -.0042 & -0.341 \\
\hline $\begin{array}{c}\text { Certificados ISO } 9000 \\
\text { e ISO } 14000\end{array}$ & $0.645^{\star}$ & $0.645^{\star}$ & $0.703^{*}$ & $0.599^{*}$ & $0.645^{\star}$ & $0.636^{*}$ & $0.645^{\star}$ \\
\hline Persona que ejerce la Gerencia & $0.645^{\star}$ & $0.645^{\star}$ & $0.703^{*}$ & $0.599^{*}$ & $0.645^{\star}$ & $0.636^{*}$ & $0.645^{\star}$ \\
\hline Programas de Productividad & $0.519^{*}$ & $0.519^{*}$ & $0.583^{*}$ & $0.525^{\star}$ & $0.519^{*}$ & $0.558^{*}$ & $0.519^{\star}$ \\
\hline
\end{tabular}

Fuente: Elaboración propia. Datos obtenidos a partir de la Encuesta analizada.

Nota: * Significativo estadísticamente al 95\%. 
- En el elemento tecnología del principal producto o servicio, se observa una leve asociación con los rasgos vinculados al componente ético dado que sólo se manifiesta correlación significativa, respecto al código de ética, hecho que podría sugerir la necesidad de desarrollar normas y principios que rijan la variable en el ámbito competitivo.

- Respecto al certificado ISO 9000 e ISO 14000, personal que ejerce la gerencia y programas de productividad, se observa que todas y cada una de las variables, poseen una asociación significativa respecto a los rasgos relacionados con el componente ético, situación que llama la atención frente al hecho de que el principal cliente, sector petrolero, demanda en forma permanente, lineamientos tendentes a lograr rasgos que garanticen la confiabilidad, calidad y excelencia necesaria para sus operaciones, contribuyendo así, a crear el compromiso de que la empresa asuma su responsabilidad, hecho que se manifiesta en su desenvolvimiento interno.

- En cuanto al elemento relacionado con la persona que ejerce la gerencia, debe recordarse que, dependiendo de su formación y visión estratégica del negocio, puede asumir los rasgos que le garanticen su rentabilidad como empresa, además de su perdurabilidad en el tiempo como organización de futuro.

\section{Gestión de la calidad en la Pequeña y Mediana Empresa Metalmecánica zuliana}

La gestión de la calidad es un proceso que se fundamenta en el conocimiento preciso de las necesidades y expectativas del cliente, compromete a todo el personal de la organización y produce una mejora en la calidad y productividad, a través del uso del Control Estadístico de Proceso, de las Técnicas del Mejoramiento Continuo de los Procesos y la Reingeniería (Berry, 1996).

De la información recolectada en las Empresas Pequeñas y Medianas del Sector Metalmecánico Zuliana, se concluye que ninguna tiene instalado un Modelo de Gestión de la Calidad propiamente dicho. Sin embargo a continuación se detallan algunos elementos rescatables, con base en los rasgos internalizados por la empresa y que reflejan la no relatividad del valor asumido: (Ver gráficos 1 y 2).

Por otro lado, prácticamente ninguna empresa (el $4,30 \%$ de las pequeñas y el $7,70 \%$ de las medianas) cuenta con programas de desarrollo tecnológico. En un mundo globalizado y en constante cambio esto se revela como una debilidad altamente significativa. La inmovilidad tecnológica, con mucha rapidez, coloca a las empresas en franca desventaja con respecto a sus competidores, sobre todo aquellas que utilizan el Mejoramiento Continuo de sus Procesos (Vázquez y Ferrer, 2001).

Esta situación produce que sólo algunas de las empresas, $13,00 \%$ de las pequeñas y $15,40 \%$ de las medianas, entreguen a su cliente un producto con una relación precio-valor de acuerdo a sus expectativas. Es indudable, que de no tomarse medida al respecto, la perdurabilidad de la mayoría 
Gestión de calidad y su dimensión ético competitiva en la pequeña y mediana empresa Ferrer, Juliana; Vázquez, José Luis; Clemenza, Caterina; Pérez, Rosario

\section{Gráfico 1 \\ Gestión de calidad. Pequeña Empresa Metalmecánica Zuliana.}

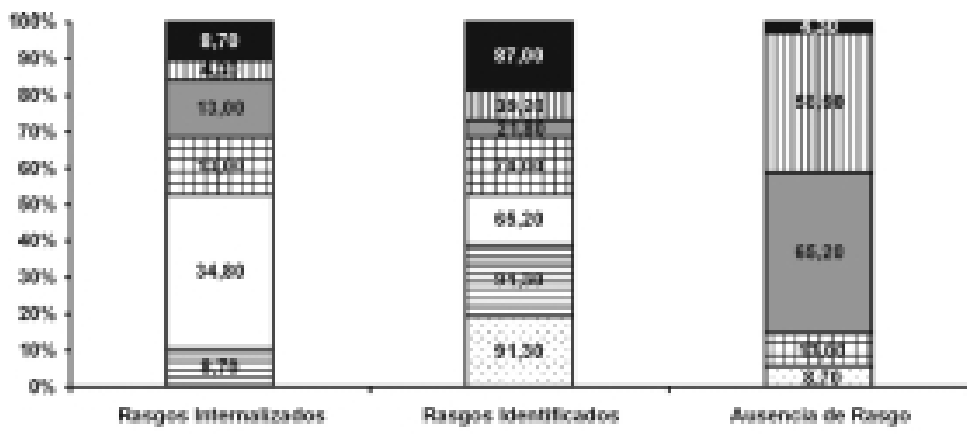

\begin{tabular}{|c|c|}
\hline & 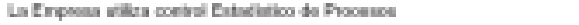 \\
\hline & 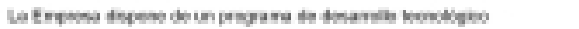 \\
\hline & 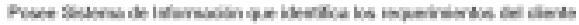 \\
\hline 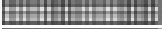 & 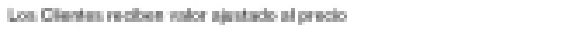 \\
\hline & 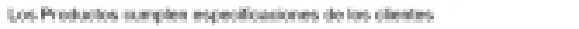 \\
\hline & Loe Provedores curples enpecifcaclones \\
\hline & 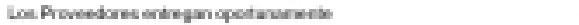 \\
\hline
\end{tabular}

Fuente: Elaboración propia. Datos obtenidos a partir de la Encuesta analizada.

\section{Gráfico 2}

\section{Gestión de Calidad. Mediana Empresa Metalmecánica Zuliana.}

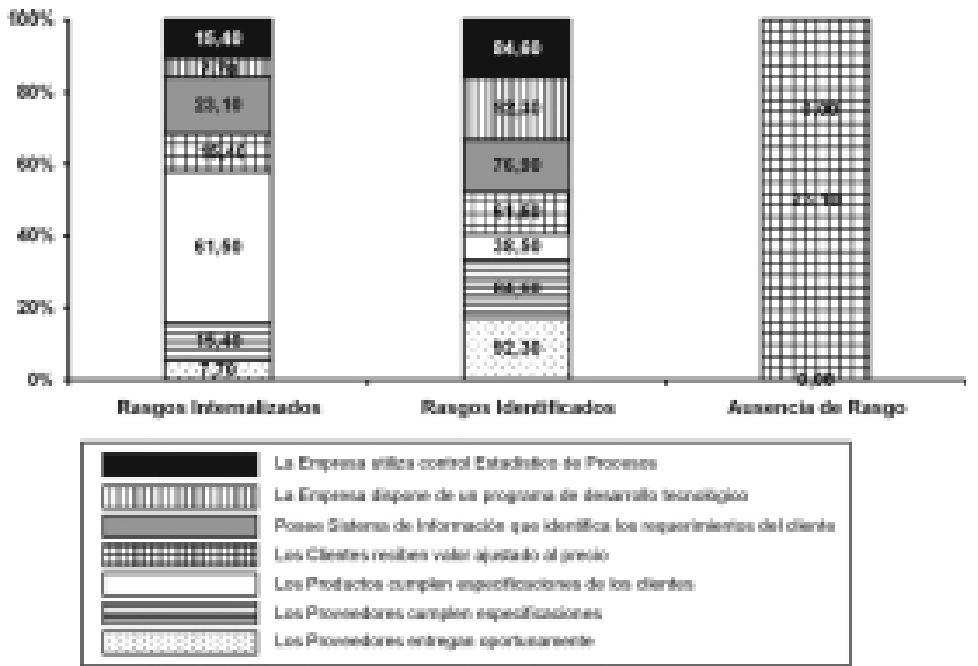

Fuente: Elaboración propia. Datos obtenidos a partir de la Encuesta analizada. 
de las empresas del sector estará en peligro (Ferrer, 2001).

Con respecto a la relación con el proveedor, condición necesaria para lograr la excelencia, la mayoría de las empresas del sector refieren enfrentar graves problemas. Se presentan pautas asociadas con proveedores poco confiables, y lo más grave, no se nota una intención por parte de las empresas del sector, para enfrentar el problema, a través de un programa de desarrollo de proveedores, tal como se aconseja en la aplicación de un Modelo de Gestión de la Calidad.

Por último, la ausencia en la mayoría de las empresas del sector, especialmente en las pequeñas $87,00 \%$, de una misión y una visión escrita, revela una falta de cohesión del personal de éstas en cuanto a la dirección que deben tomar para alcanzar sus objetivos estratégicos. Además si se toma en consideración, el desconocimiento por parte del personal de las acciones que deben acometerse en conjunto, a fin de aumentar la calidad y productividad en la empresa, la situación se torna ciertamente vulnerable frente a las exigencias del entorno global.

\section{A modo de conclusión}

La calidad como valor organizacional y la gestión de la calidad como una herramienta, son aceptadas por la mayoría de los gerentes como forma de enfrentar situaciones de cambio en un mundo de aceleradas transformaciones, donde la visión de la calidad se convierte en un elemento ético competitivo clave para transitar en el contexto globalizado. Las cuestiones éticas en las organizaciones no pueden tratarse como si fueran proble- mas de simples optimización de decisiones; son visiones frente a valores en juego, considerados como un imperativo para el tránsito congruente de las empresas a través de espacios no delineados. Cualquier estudio tendente a propiciar un diálogo entre el componente ético y la competitividad debe tener como norte la búsqueda de una interacción estructurada y concertada entre clientes internos y externos, en la necesidad de internalizar entre ellos un conjunto de valores organizacionales, que al ser aceptados, posicionan a la empresa de manera ventajosa frente a sus competidores. Tal situación se manifiesta en el valor calidad, por cuanto su aplicación, dentro de la filosofía de la empresa, representa un elemento competitivo por excelencia para enfrentar la incertidumbre que generan los cambios en el mercado.

En las últimas décadas ha habido un creciente interés por las pequeñas y medianas empresas, como vínculo idóneo para lograr el crecimiento en economías dentro del plano mundial. Hoy día la naturaleza de las relaciones y no el tamaño, determinan el factor competitivo, logrando una interacción viable entre clientes internos y externos, a través de la gestión de la calidad.

En el contexto venezolano, la pequeña y mediana empresa ha tenido un papel crucial como proveedor de ciertos sectores, especialmente el petrolero, además de haber garantizado por varias décadas, niveles de empleo y bienestar para el país. Sin embargo, en el contexto empresarial de las pequeñas y medianas empresas se observan deficiencias gerenciales, organizativas y técnicas, de las cuales no escapa la Metalmecánica 
Zuliana, además de gran dificultad para los procesos de mejora en cuanto a productividad y cambios tecnológicos y organizativos.

Los resultados arrojados en el presente artículo demuestran estas afirmaciones, evidenciadas en que las empresas refieren una alta capacidad ociosa, explicada no sólo por la contracción de la demanda del sector, sino por una baja eficacia de sus procesos productivos; igualmente se revela un bajo desarrollo tecnológico, poca capacidad de diversificación y escasos programas de productividad; elementos fundamentales para enfrentar con éxito mercados competitivos inciertos.

El estudio mostró que existe alta correlación entre la competitividad y algunos elementos éticos ligados al rasgo calidad, arrojando un valor para la Rho de Spearman de 0,89 . Esto revela una asociación positiva entre la Competitividad de las Empresas Pequeñas y Medianas del Sector Metalmecánico Zuliano y Elementos Éticos relacionados a la Calidad.

Igualmente es interesante resaltar que ninguna empresa del sector tiene instalado un Modelo de Gestión de la Calidad propiamente dicho, que se evidencia en la ausencia manifiesta de los elementos característicos de éste: Sistema de Información orientados al mercado, Control Estadístico de Proceso, Programa de Desarrollo Tecnológico, procesos orientados hacia el cliente, Relación Precio-Valor cónsonas con las expectativas del cliente y sobre todo, herramientas de orden estratégico que le permitan enfrentar las condiciones de incertidumbre por las que transitan inadvertidamente, al incorporarse a mercados globalizados, espe- cialmente representados por el mercado petrolero mundial.

En este sentido se hace necesario adelantar acciones de mejora dentro de cada empresa, compatible con el modo de vida de cada estrato, lo que incluye entender aspectos clave como capacidad para optimar los métodos de mejora continua; enfocarse en la calidad; abandonar la complejidad y acogerse a la simplicidad y sobre todo, instalar nuevas formas de liderazgo que garanticen la internalización de la calidad como filosofía de vida, en la garantía de constituirse en el elemento competitivo para la consolidación de las empresas del sector.

\section{Bibliografía Citada}

\section{Berry, Thomas (1996). Cómo gerenciar la transformación hacia la Calidad Total. Mc Graw Hill. Colombia.}

Bradford, Lawrence J.; Albrecht, Karl (1992). Calidad del Servicio. Editorial Díaz de Santos, S. A. Colombia.

Cantun Delgado, Humberto (1997). Desarrollo de una Cultura de Calidad. 1era. Edición. Mc Graw-Hill Interamericana. Editores S.A. de C.V. México.

Ciampa, C. (1992). Calidad del Servicio al Cliente. Editorial Mc Graw Hill. México.

Clemenza, Caterina; Urbina, Angel; Rodríguez, Rosmary; Roncacio, Alba y Roncacio, Liliana (1997). La pequeña y mediana industria metalmecánica ante el proceso de apertura petrolera. En: Revista Venezolana de Gerencia. Año 2, № 4. LUZ. Maracaibo, Venezuela. Pp. 193-211.

Clery A., Arturo G. (2001). La calidad total como una estrategia competitiva aplicada a una empresa de servicios de instalaciones eléctricas y 
telecomunicaciones.

Http://www.monografias.com/

trabajos6/lacali/lacali.shtml\#calidad.

[03-10-01].

Cobra, Marcos (2000). Marketing de Servicios. 2da Edición. Mc Graw Hill. Colombia.

Cobra, Marcos; Zwarg, Flávio (1991). Marketing de Servicios. México.

Cortina, Adela (1985). Razón Comunicativa y Responsabilidad Solidaria. Editorial Tecnos. España.

Cortina, Adela (1990). Ética sin Moral. Editorial Tecnos. España.

Cortina, Adela (1992). Concepciones de la Ética. Editorial Trotta. Madrid.

Cortina, Adela (1993). Ética Aplicada y Democracia Radical. Editorial Tecnos. España.

Cortina, Adela (1996). Ética de la Empresa. Editorial Trotta 2da Edición. Madrid.

Cortina, Adela (1998). Razón Práctica. En Cortina, Adela (1998). En 10 Palabras en Ética. Editorial Verbo Divino. España, pp. 26-37.

Cortina, Adela; Castiñeira Ángel; Conill, Jesús; Domingo, Agustín; García, Domingo; Lozano, José Félix y Lozano Josep María (1997). Rentabilidad de la Ética para la Empresa. Fundación Argentina. Visor Pis. España.

Cortina, Adela; Conill, Jesús (1998). Democracia Participativa y Sociedad Civil. Una Ética Empresarial. Fundación Social Siglo del Hombre Editores. Colombia.

Deming, W. Edwards. (1986). Out of the Crisis. MIT Press. Cambridge, MA.

Eiglier, Pierre; Langeard, Eric, (1989). Servucción. El Marketing de Servicios. Mc Graw-Hill/Interamericana de España, S. A., España-Madrid.

Etkin, Jorge (1996). La empresa competitiva. Grandeza y decadencia. Un cambio hacia una organización viable. Mc Graw-Hill. Chile.

Fea G., Ugo (1993). Competitividad es Calidad Total. Manual para salir de la Crisis y Generar Empleo. Creación y Desarrollo Empresarial. Edición original Marcombo, S.A., 2da. Edición 1995. Ediciones Alfaomega, S.A. Barcelona-España.

Fedeindustria, (1996). Elementos para una Política Tecnológica Nacional. Sector Industrial. Caracas-Venezuela.

Ferrer, Juliana (2001). Presencia del Componente Ético en Sectores de Actividad Industrial. Ediciones Astro Data, S. A. Maracaibo, Venezuela.

Ferrer, Juliana; Clemenza, Caterina; Vázquez, José (1999). Estrategias competitivas. Un aporte a la búsqueda y consolidación del sector metalúrgico y metalmecánico de la región zuliana. En: Revista Venezolana de Gerencia. Año $4 \mathrm{~N}^{\circ}$ 9. Vicerrectorado Académico-LUZ. Venezuela. Pp. 71-91.

Gitlow, Howard S. (1991). Planificando para la Calidad la Productividad y una Posición Competitiva. Ventura Ediciones, S.A. México.

Hammer, Michael y Champy, James (1997). Reingeniería . Grupo Editorial Norma. Colombia.

Harrington H., James (1988). Cómo incrementar la Calidad Productividad en su Empresa. Mc Graw Hill. México.

Hernández F., Lissette (1998). Los Estudios de Competitividad en América Latina y Venezuela. Trabajo de Ascenso para optar a la categoría de Profesor Agregado. FCES-Escuela de Economía-LUZ. Venezuela.

Hit, Michael; Ireland, Deiane; Hoskisson, Robert (1999). Administración Estratégica. Conceptos Competitividad y Globalización. International Thomson Editores. México. 
Horovitz, Jacques (1991). La Calidad del Servicio a la Conquista del Cliente . Editorial McGraw Hill. Madrid.

Juran, Joseph M. Juran y el Liderazgo para la Calidad (1990). Un Manual para Directivos. Ediciones Díaz de Santos, S. A. España.

Katz, Jorge (1983). El Escenario Tecnológico Latinoamericano en el Campo Metalmecánico. Revista CEPAL, Abril. Chile.

Kotler, Philip; Armstrong, Gary (1996). Mercadotecnia. Editorial Mc Graw Hill. México.

Lanusse Storni, José A. (2001). Las Reformas Estructurales y las Micro, Pequeñas y Medianas Empresas: Una Agenda Posible. En: Revista Contribuciones. Vol. 1, № 1/2001. Konrad Adenauer - Stiftung. Pp. 67-89.

Lovelock, Christopher (1997). Mercadotecnia de Servicios. 3ra. Edición. PrenticeHall Hispanoamericana, S. A., México.

Oficina Central de Estadística e Informática (O.C.E.I.) (1997). Sexta Encuesta Industrial-Estado Zulia. Período 19891994. Caracas.

Pilleti, Giancarlo (1999). Foro Plan de Desarrollo del Sector Metalmecánico y Metalúrgico en el Estado Zulia. Memorias del Evento. Maracaibo-Venezuela.

Pizani, Leonardo (1994). La Hora de la Pequeña y Mediana Industria. En: Revista del Centro Nacional de Calidad y Productividad de la Pequeña y Mediana Industria (CEPROPYMI). Año 1, No. 1. Octubre - noviembre. Pp. 1634. Caracas.

Romero, Jenny; Acosta, Ana; Bohórquez, Elizabeth (1997). Situación de la Pe- queña y Mediana Industria (PyMI) en la Región Zuliana. Período 1989-1994. En: Revista de Ciencias Sociales.FCES-LUZ., Nueva Época, Vol. III, N 3 . Venezuela. Pp. 207-229.

Romero, Jenny; Acosta, Ana; Bohórquez, Elizabeth (1998). ¿Es Competitiva la Pequeña y Mediana Industria de la Región Zuliana? En: Revista Venezolana de Gerencia. Año 3, № 5 . Vicerrectorado Académico-LUZ. Venezuela. Páginas: 91-109.

Rosales L., Ramón (1996). Estrategias Gerenciales para la Pequeña y Mediana Empresa. Fundación Polar. Ediciones IESA. Caracas.

Scholtes, Peter R. (1993) Total Quality or Perfomance Appraisal: Choose one. Http://www.iqpic.org/per.htm. [11-08-01].

Serieyx, Herve (1991). El Desprecio Cero. Hacia la Calidad Total. McGraw Hill. España.

Shaw, J. (1997). El Cliente Quiere Calidad. Editorial Prentice Hall. México.

Shewhart, W. A. (1939). Aplication of Statistical Method in Mass Production. Proceeding of the Industrial Statistic Conference Held at Massachusetts Institute of Technology. Pitman Publishing, Nueva York.

Singh, Sarv (1998). Control de calidad total. Claves, metodologías y administración para el éxito. Mc Graw Hill. México.

Vázquez, José; Ferrer, Juliana (2001). Elementos claves de la gestión de la calidad en la nueva lógica del mundo contemporáneo. En: TELOS Revista de Estudios Interdisciplinarios Universidad "Rafael Belloso Chacín". URBE, Maracaibo, Venezuela. Pp. 92-107. 